\title{
Complete Response of Recurrent Small Cell Carcinoma of the Uterine Cervix to Paclitaxel, Carboplatin, and Bevacizumab Combination Therapy
}

\author{
Yuri Nakao Satoshi Tamauchi Nobuhisa Yoshikawa Shiro Suzuki \\ Hiroaki Kajiyama Fumitaka Kikkawa \\ Department of Obstetrics and Gynecology, Nagoya University Graduate School of \\ Medicine, Nagoya, Japan
}

\section{Keywords}

Small cell carcinoma $\cdot$ Cervical cancer $\cdot$ Bevacizumab

\begin{abstract}
We report a case of recurrent small cell carcinoma of the uterine cervix that showed a complete response to paclitaxel, carboplatin, and bevacizumab ( $\mathrm{TC}+\mathrm{Bev})$ combination therapy. Small cell carcinoma of the uterine cervix is extremely rare, with an incidence of only $1.3 \%$ in Japan, and a poor outcome. The patient was a 62-year-old woman with a chief complaint of irregular vaginal bleeding. Magnetic resonance imaging showed a $10-\mathrm{cm}$ irregular mass from the uterine corpus's posterior wall to the cervix. Abdominal total hysterectomy, bilateral salpingo-oophorectomy, and pelvic lymph node sampling were performed for suspected uterine sarcoma. Histopathological findings revealed small cell carcinoma with lymph node metastasis. Although 6 cycles of etoposide + cisplatin were performed, para-aortic lymph node recurrence was found 3 months after chemotherapy. Subsequently, the patient received 8 cycles of $\mathrm{TC}+\mathrm{Bev}$, which eliminated the metastases. The patient is currently alive at 24 months.

\section{Introduction}

Small cell carcinoma of the uterine cervix is a very aggressive neuroendocrine tumor that accounts for only $1.3 \%$ of cervical cancers [1]. Lymph node metastasis and distant metastasis can occur at an early stage with poor outcomes [2,3]. The 4-year overall survival (OS) was reported as follows: FIGO stage IB1, 63\%; stage IB2, 67\%; stage IIB, 30\%; stage IIIB, 29\%; and 

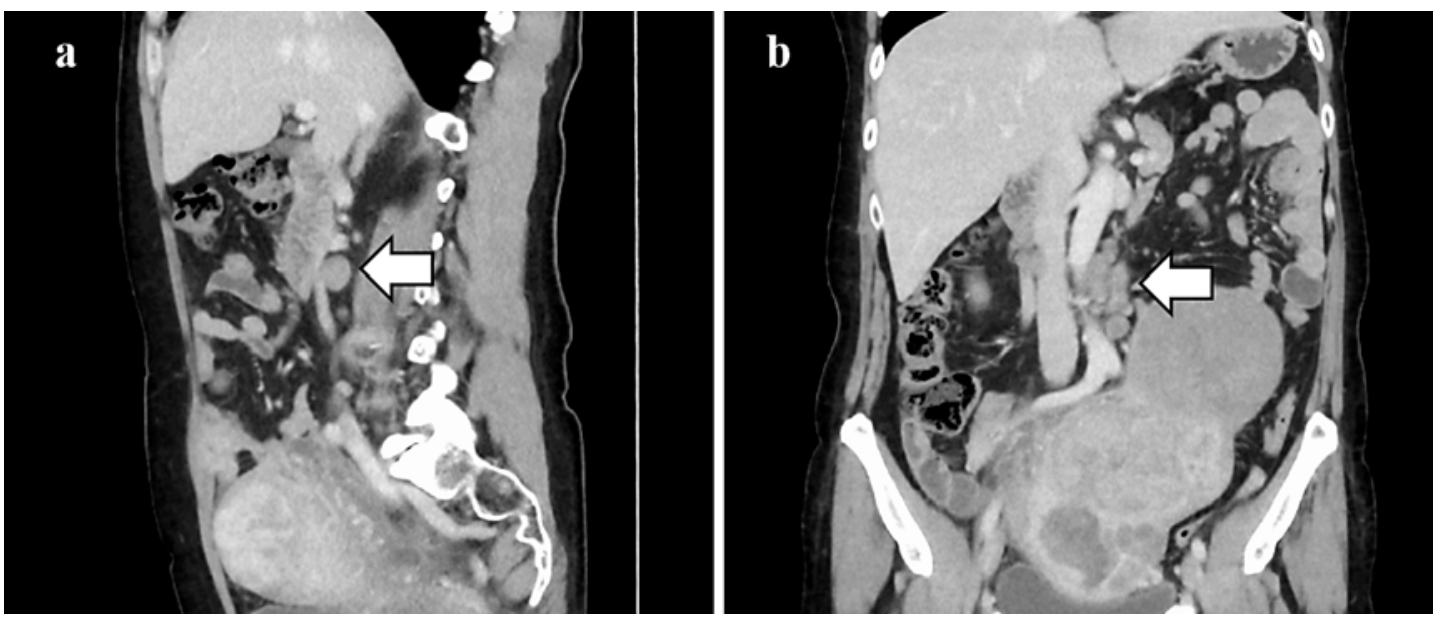

Fig. 1. Computed tomography findings at the first diagnosis. Para-aortic lymph nodes were enlarged (arrows). a Sagittal plane. b Coronal plane.

stage IVB, 25\% [4]. The optimal initial therapeutic approach has not been identified; therefore, we often follow the treatment regimens for small cell lung cancer, such as cisplatin and etoposide [5, 6]. However, it is a bigger challenge to treat recurrent small cell carcinoma, and no treatment consensus has been reached to date.

Bevacizumab is a humanized monoclonal antibody that targets the vascular endothelial growth factor, which promotes angiogenesis [7, 8]. In Japan, the National Health Insurance program has suggested the treatment of advanced recurrent uterine cervical carcinoma with a combination of bevacizumab and paclitaxel since 2016, which was based on the GOG 240 study [9]. In that study, the addition of bevacizumab to combination chemotherapy for patients with recurrent metastatic cervical cancer was associated with an improved median OS. Bevacizumab is known to be efficient for patients with recurrent cervical cancer $[9,10]$. Combination therapy with bevacizumab could also be adapted for the treatment of small cell carcinoma, but the number of small cell carcinoma cases is insufficient to establish an effective treatment.

Here, we present a case of small cell carcinoma of the uterine cervix in a 62-year-old female in which para-aortic lymph node recurrence was completely eliminated by paclitaxel, carboplatin, and bevacizumab (TC + Bev) combination therapy.

\section{Case Report}

The patient was a 62-year-old woman with a history of five pregnancies and four childbirths. She first noted irregular vaginal bleeding, which persisted for 3 months. Magnetic resonance imaging showed a $10 \times 9 \times 8 \mathrm{~cm}$, irregular, poorly defined mass growing from the posterior wall of the uterine corpus to the cervix and high intensity on T2-weighted images, which suggested uterine sarcoma. When the patient was first seen at our department, an enlarged left supraclavicular lymph node was recognized. Laboratory tests showed that her white blood cell count was $9,000 / \mu \mathrm{L}$, hemoglobin was $11.2 \mathrm{~g} / \mathrm{dL}$, the platelet count was $549 \times 10^{3} / \mu \mathrm{L}$, and lactate dehydrogenase was $723 \mathrm{IU} / \mathrm{L}$. Regarding tumor markers, squamous cell carcinoma antigen was $0.7 \mathrm{ng} / \mathrm{mL}$, carbohydrate antigen $19-9$ was $7 \mathrm{U} / \mathrm{mL}$, and carbohydrate antigen 125 was $54.9 \mathrm{U} / \mathrm{mL}$. Cervical and endometrial smears were performed, which 

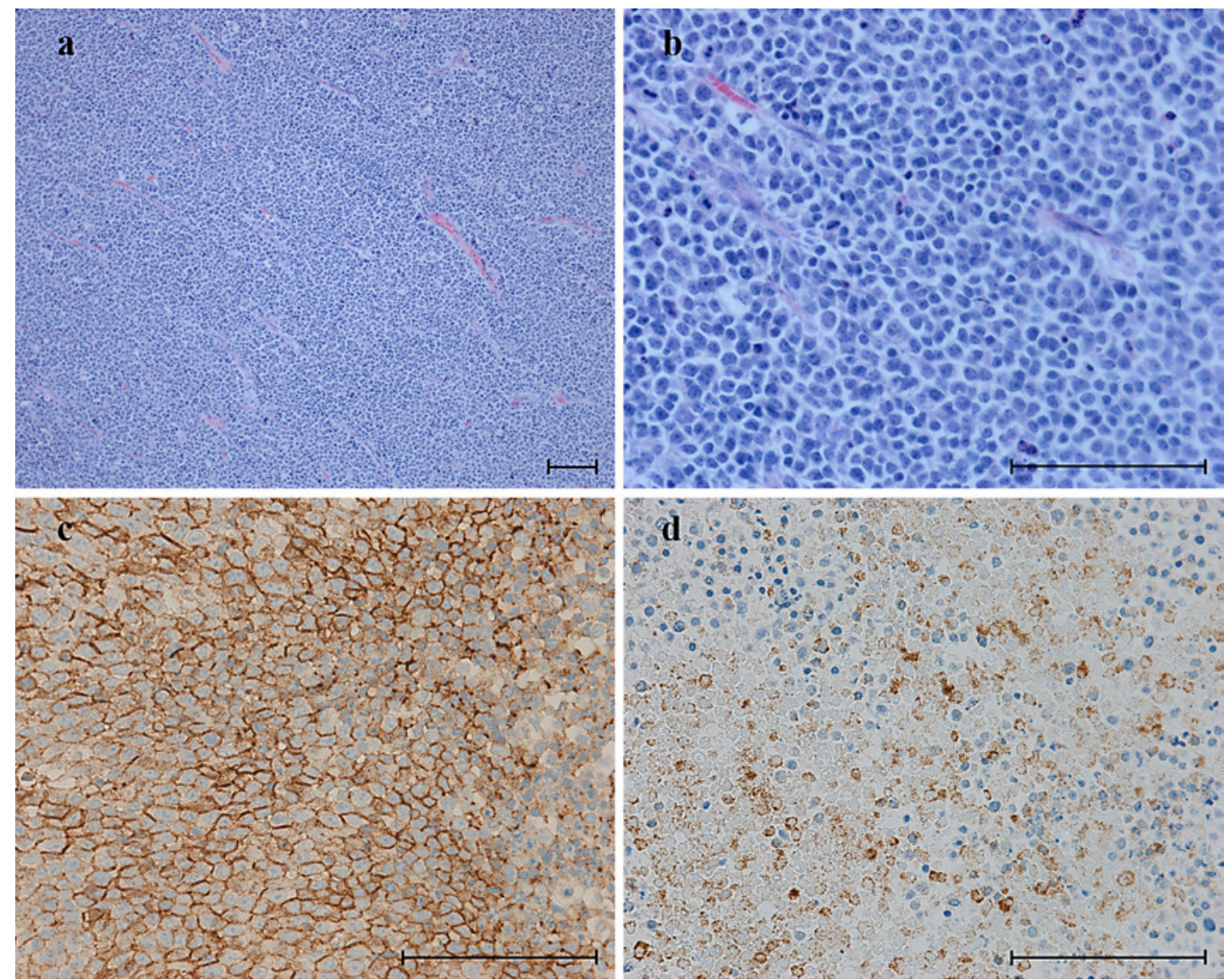

Fig. 2. Histological findings in the uterine cervix. a, b A prevalent solid pattern composed of small cells with hyperchromatic nuclei may be seen. HE. $\mathbf{a} \times 100$. $\mathbf{b} \times 400$. Scale bars, $100 \mu \mathrm{m}$. $\mathbf{c}$ Immunohistochemical staining for CD56. $\times 400$. Scale bar, $100 \mu \mathrm{m}$. d Immunohistochemical staining for chromogranin A. $\times 400$. Scale bar, 100 $\mu \mathrm{m}$.

revealed no malignancy. Computed tomography (CT) scans showed an enlarged uterus and enlarged lymph nodes, including the para-aortic lymph nodes (Fig. 1). Uterine sarcoma was primarily suspected, and the patient underwent abdominal total hysterectomy, bilateral salpingo-oophorectomy, and pelvic lymph node sampling.

Macroscopic examination of a surgical specimen revealed a bulky tumor from the uterine cervix to part of the corpus. Microscopic examination (Fig. 2a, b) revealed a prevalent solid pattern composed of small cells with hyperchromatic nuclei. On immunohistochemical analysis, CD56 (Fig. 2c) and chromogranin A (Fig. 2d), which are specific to neuroendocrine tumors, were positive. However, mesenchymal markers such as desmin, $\alpha$-SMA, or vimentin were not positive. Thus, the pathological diagnosis was FIGO stage IVB small cell carcinoma of the uterine cervix (pT2bN1M1). Distant metastasis was assessed to the left supraclavicular lymph node. The level of neuron-specific enolase (NSE) was determined using serum on the day of surgery, and it was found to be $58.6 \mathrm{ng} / \mathrm{mL}$. Because the lesions remained in the paraaortic lymph nodes, she was started on 6 cycles of etoposide + cisplatin (EP) therapy (E: 100 $\mathrm{mg} / \mathrm{m}^{2}$, days $1-3 ; \mathrm{P}: 80 \mathrm{mg} / \mathrm{m}^{2}$, day 1 ), based on the treatment for small cell lung cancer, which she tolerated well. After 6 cycles of EP, CT scans revealed that the para-aortic lymph node lesions were decreasing in size, which we assessed as a partial response to the treatment and then followed up at stated intervals. At 3 months after chemotherapy, her NSE level had 

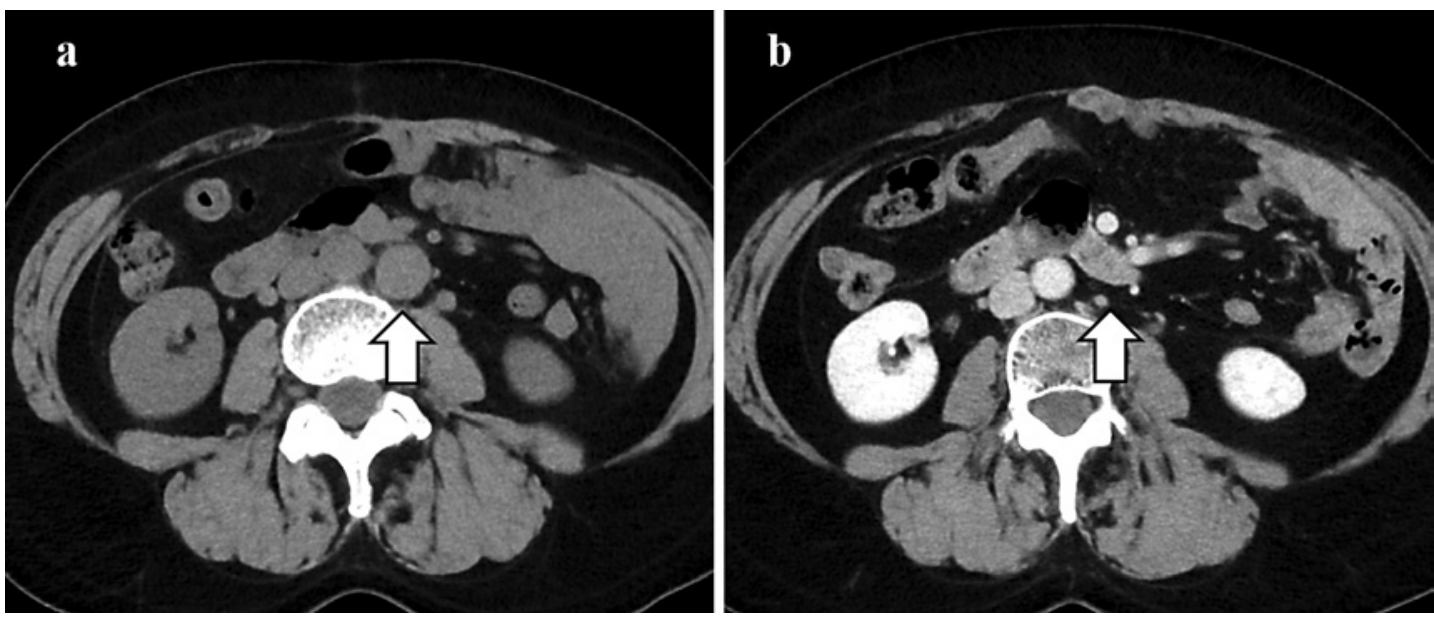

Fig. 3. Computed tomography (CT) findings. a The size of the para-aortic lymph nodes was increased (arrow) at 3 months after etoposide + cisplatin therapy. Because of the lowering of renal function, only plain CT was successful. b The para-aortic lymph nodes have diminished (arrow) at 18 months after paclitaxel, carboplatin, and bevacizumab combination therapy.

increased, and CT scans showed an increase in the size of the para-aortic lymph node lesions (Fig. 3a), for which recurrence was diagnosed.

$\mathrm{TC}+\mathrm{Bev}$ was administered (T: $180 \mathrm{mg} / \mathrm{m}^{2} ; \mathrm{C}: \mathrm{AUC}=6 ; \mathrm{Bev}: 15 \mathrm{mg} / \mathrm{kg}$ ). She showed some adverse chemotherapy effects, including pancytopenia, hypertension, and urine protein. After 6 cycles of chemotherapy, the lesions had partially responded to the treatment, and after 2 more cycles, they had completely responded. In total, 8 cycles of chemotherapy were administered. CT scans revealed that the para-aortic lymph node lesions had diminished (Fig. $3 \mathrm{~b}$ ) and the patient's NSE level had normalized. The patient is currently alive at 24 months after the TC + Bev therapy was completed, and no sign of recurrence has been observed.

\section{Discussion}

Small cell carcinoma is a fast-growing tumor with neuroendocrine differentiation that usually occurs in the lungs. In Japan, small cell carcinoma of the uterine cervix is very rare, accounting for only $1.3 \%$ of uterine cervical carcinomas [1]; therefore, it is difficult to conduct a prospective study. The poor prognosis is because of early lymph node development, distant organ metastases, and vascular invasion, which are risk factors for recurrence [2,3]. The first symptom is usually postmenopausal bleeding. Radical hysterectomy with adjuvant chemotherapy or chemoradiation has improved survival $[2,11]$. However, because no effective therapy has been established, we often follow the treatment regimens for small cell lung cancer or squamous cell carcinoma of the uterine cervix, which include cisplatin and etoposide $[5,6]$. It is more difficult to treat recurrent small cell carcinoma, and no consensus has been reached.

The GOG 240 study revealed that bevacizumab along with chemotherapy (paclitaxel + cisplatin [TP] or paclitaxel + topotecan) prolonged OS with recurrent/persistent or advanced cervical cancer. The researchers randomly assigned 452 patients to chemotherapy with or without bevacizumab. Chemotherapy with bevacizumab was associated with increased OS 
and increased progression-free survival. The response rate was markedly higher among those patients who received bevacizumab than among those who did not (relative probability of a response, 1.35; 95\% CI, 1.08-1.68; $p=0.008$ ) [9]. On the basis of that study, Godoy-Ortiz et al. [10] compared the efficacy and safety of chemotherapy with bevacizumab with their clinical patients and demonstrated the efficacy of bevacizumab as part of first-line treatment for advanced cervical cancer.

Frumovitz et al. [12] retrospectively analyzed 34 women with recurrent small cell carcinoma of the uterine cervix who received chemotherapy as the primary therapy; 13 received topotecan, paclitaxel, and bevacizumab for recurrence, and 21 received another chemotherapy that was primarily platinum with or without a taxane. The median progressionfree survival was 7.8 months for treatment with topotecan, paclitaxel, and bevacizumab and 4.0 months for the other group (HR, $0.21 ; 95 \% \mathrm{CI}, 0.09-0.54 ; p=0.001$ ). The median OS was 9.7 months for treatment with topotecan, paclitaxel, and bevacizumab and 9.4 months for the other group (HR, $0.53 ; 95 \% \mathrm{CI}, 0.23-1.22, p=0.13$ ). In the topotecan, paclitaxel, and bevacizumab group, 3 patients (23\%) showed a complete response. To our knowledge, no studies except those described have focused on the effectiveness of combined chemotherapy with bevacizumab for recurrent small cell carcinoma [11].

In Japan, combined chemotherapy with topotecan is not widely used, and TC or TP is more common for gynecological cancers. Hirasawa et al. [13] reported on a patient with recurrent refractory small cell carcinoma of the uterine cervix for whom combined therapy with TP + Bev was effective. After postoperative adjuvant chemotherapy with EP and radiation therapy, multiple liver metastases were detected, which indicated refractory disease. That patient received $\mathrm{TP}+\mathrm{Bev}$, and is currently alive at 16 months after recurrence was detected.

In our case, we administered $\mathrm{TC}+\mathrm{Bev}$ to treat recurrent small cell carcinoma of the uterine cervix. TC is a relevant regimen, and we are accustomed to TC use in terms of adverse effects or management. The JCOG0505 trial showed that TC was not inferior to TP in metastatic or recurrent cervical cancer [14]. After 6 cycles of this combined therapy, the size of the para-aortic lymph nodes was partially reduced, and 2 more cycles achieved a complete response. At present, the para-aortic lesions are no longer detectable.

A limited number of case reports have suggested the effectiveness of bevacizumab, but no studies have reported that bevacizumab + TC or TP prolonged the prognosis of small cell carcinoma of the uterine cervix. Bevacizumab therapy should be considered as a potentially beneficial therapy for recurrent small cell carcinoma of the uterine cervix.

\section{Acknowledgements}

We sincerely thank all the members of the Department of Obstetrics and Gynecology, Nagoya University Hospital.

\section{Statement of Ethics}

To report this case, we obtained written consent from the patient.

\section{Disclosure Statement}

The authors declare that they have no conflicts of interest. 


\section{Funding Sources}

We did not receive any funding for this report.

\section{Author Contributions}

Y.N. wrote this manuscript; S.T. and N.Y. revised the manuscript; S.S. is the attending doctor of this patient; S.S., H.K., and F.K. supervised this article.

\section{References}

1 Nagase S, Ohta T, Takahashi F, Enomoto T; 2017 Committee on Gynecologic Oncology of the Japan Society of Obstetrics and Gynecology. Annual report of the Committee on Gynecologic Oncology, the Japan Society of Obstetrics and Gynecology: annual patients report for 2015 and annual treatment report for 2010. J Obstet Gynaecol Res. 2019;45(2):289-98.

2 Cohen JG, Kapp DS, Shin JY, Urban R, Sherman AE, Chen LM, et al. Small cell carcinoma of the cervix: treatment and survival outcomes of 188 patients. Am J Obstet Gynecol. 2010;203(4):347.e1-6.

3 Viswanathan AN, Deavers MT, Jhingran A, Ramirez PT, Levenback C, Eifel PJ. Small cell neuroendocrine carcinoma of the cervix: outcome and patterns of recurrence. Gynecol Oncol. 2004;93(1):27-33.

4 Kuji S, Hirashima Y, Nakayama H, Nishio S, Otsuki T, Nagamitsu Y, et al. Diagnosis, clinicopathologic features, treatment, and prognosis of small cell carcinoma of the uterine cervix; Kansai Clinical Oncology Group/Intergroup study in Japan. Gynecol Oncol. 2013;129(3):522-7.

5 Gardner GJ, Reidy-Lagunes D, Gehrig PA. Neuroendocrine tumors of the gynecologic tract: a Society of Gynecologic Oncology (SGO) clinical document. Gynecol Oncol. 2011;122(1):190-8.

6 Satoh T, Takei Y, Treilleux I, Devouassoux-Shisheboran M, Ledermann J, Viswanathan AN, et al. Gynecologic Cancer InterGroup (GCIG) consensus review for small cell carcinoma of the cervix. Int J Gynecol Cancer. 2014; 24(9 Suppl 3):S102-8.

7 Leung DW, Cachianes G, Kuang WJ, Goeddel DV, Ferrara N. Vascular endothelial growth factor is a secreted angiogenic mitogen. Science. 1989;246(4935):1306-9.

8 Ferrara N, Hillan KJ, Gerber HP, Novotny W. Discovery and development of bevacizumab, an anti-VEGF antibody for treating cancer. Nat Rev Drug Discov. 2004;3(5):391-400.

9 Tewari KS, Sill MW, Long HJ 3rd, Penson RT, Huang H, Ramondetta LM, et al. Improved survival with bevacizumab in advanced cervical cancer. N Engl J Med. 2014;370(8):734-43.

10 Godoy-Ortiz A, Plata Y, Alcaide J, Galeote A, Pajares B, Saez E, et al. Bevacizumab for recurrent, persistent or advanced cervical cancer: reproducibility of GOG 240 study results in "real world" patients. Clin Transl Oncol. 2018;20(7):922-7.

11 Monk BJ, Tewari KS, Koh WJ. Multimodality therapy for locally advanced cervical carcinoma: state of the art and future directions. J Clin Oncol. 2007;25(20):2952-65.

12 Frumovitz M, Munsell MF, Burzawa JK, Byers LA, Ramalingam P, Brown J, et al. Combination therapy with topotecan, paclitaxel, and bevacizumab improves progression-free survival in recurrent small cell neuroendocrine carcinoma of the cervix. Gynecol Oncol. 2017;144(1):46-50.

13 Hirasawa T, Kajiwara H, Machida H, Iida T, Ikeda M, Shida M, et al. Recurrent small cell carcinoma of the uterine cervix responding to combined therapy with paclitaxel, cisplatin, and bevacizumab: a case report. Tokai J Exp Clin Med. 2018;43(3):81-4.

14 Nishio S, Kitagawa R, Shibata T, Yoshikawa H, Konishi I. Prognostic factors from a randomized phase III trial of paclitaxel and carboplatin versus paclitaxel and cisplatin in metastatic or recurrent cervical cancer: Japan Clinical Oncology Group (JCOG) trial: JCOG0505-S1. Cancer Chemother Pharmacol. 2016;78(4):785-90. 